\section{Editorial procedures reviewed}

The advantages and disadvantages of the peer review process have long been debated (see, for example, Nature 422, 259-261 (2003)), although in our view no entirely satisfactory alternative has yet been found. With the advent of new journals, each with their unique approach to the review process, we feel the time is ripe to expand on the rhyme and reason behind the current review process at Nature Cell Biology.

Dramatic technological progress, and recent increases in research funding and the number of researchers, have conspired to accelerate the speed with which key biological questions are answered. As a direct consequence, the rate and volume of manuscript submissions has risen proportionally. Consider how long it took you to survey the new literature twenty or even ten years ago. Now compare this with today, when it is likely that more often than not, you would struggle even to keep up with recent literature that is directly relevant to your work, let alone having enough time to read primary papers from areas tangential your own. For example, a comprehensive literature search on p53 would have identified approximately 700 papers each year in the early ' 90 s, 2,500 papers per year by the late ' 90 s and a staggering 3,300 papers in the new millennium.

At this journal, we publish approximately one tenth of the submitted manuscripts. This rate is determined both by the need to prevent the journal bursting at its seams, but importantly also by the stringent criteria our referees and editors impose on manuscripts. We see it as an important role for the more general journals (and NCB's purview is the whole of cell biology) to act as a filter, publishing papers that provide a truly exceptional conceptual advance of broad interest to the wider community, as well as having a foundation of well-developed, solid data. The majority of manuscripts are rejected without review after in-depth editorial analysis. At this stage, the criteria applied are both scientific and editorial. A large number of manuscripts are returned to authors on the basis that they simply do not provide a 'sufficiently striking advance', a justification that may cause frustration to some who feel that there must be another reason underlying the decision and that this is not a justifiable basis for a rejection. We would like to emphasize, however, that such a decision in no way questions the strength of the work nor its importance for researchers in the field. Decision letters do not always justify the reasoning of the editors in great detail and this is understandably a cause for concern on occasion. The reason for such brevity is simply that we aim to prioritize editorial time to allow for the informed and comprehensive assessment of each manuscript in its entirety. Each manuscript is discussed at length among a team of editors with active research experience in a diverse set of fields that collectively span broad areas of cell biology. As necessary, we will also seek informal advice from experts in the field. In light of these discussions, it is often the regrettable conclusion that the work, although important, is primarily of interest to a particular research community and would therefore be better suited to a journal that targets that particular audience.

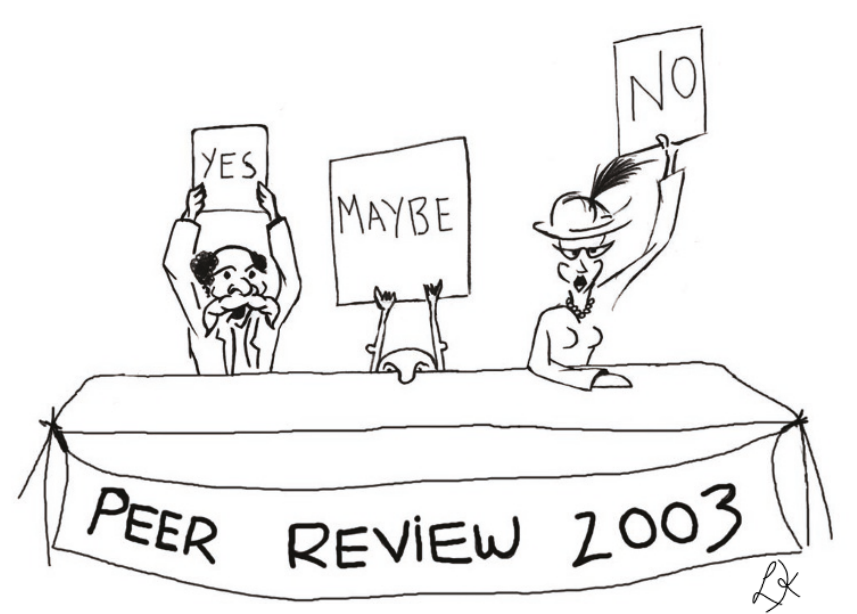

At the same time, editorial decisions are often made to return manuscripts to the authors in cases where it is our informed opinion that the work, although in principle important and of interest to a wide audience, is too premature a dataset to be published without major additional work. The aim of such decisions is to avoid futile review cycles that ultimately waste valuable referee time as well as leading to unnecessary delays that can compromize the competitiveness of the submitted data. A common misconception is that 'getting your foot in the door' safeguards a paper against being scooped by related work. This is not the case, however, because related work published elsewhere is taken into consideration upon resubmission of any manuscript that required substantive revision. The lure of an earlier publication date may also encourage premature submission. However, in fairness to everyone we reset publication dates when substantive revision is necessary.

\section{GRINDING THE AXE OR SCRATCHING THEIR BACKS?}

Nature Cell Biology, along with other Nature research journals, is fairly unique in not having an explicit editorial board. This system has served the Nature journals well over many years and we stand by it: this is not because we favour autocratic editorial-based decision-making. Rather, we feel that a finite board has the potential to restrict the decision-making to one or two individuals from each sub-discipline. An obvious concern with this system is that, on occasion, it may be a challenge to dissociate political motivation from scientific evaluation. Furthermore, board members are inevitably swamped with manuscripts while having research programmes to run at the same time, which undoubtedly puts temporal constraints on many a decision. We work on the principle that our team of editors, experts in a diverse and complementary set of cell biological areas, are best placed to administer an independent editorial opinion. Naturally, superimposed on this, we do consult with expert researchers on all experimental details throughout the peer-review process. 
We recognize the competitive nature of many disciplines in cell biology, and are committed to ensuring a rapid and constructive peerreview process. As a standard, we ask reviewers to complete their report within 1-2 weeks, a timeframe that we feel avoids compromising quality for speed. Nevertheless, a leading cell biologist holds the view that even within this timeframe, "this unfortunate modern practice encourages - indeed, almost guarantees - superficiality of reviewing, and thus that the papers entering the literature will be of lower quality".

Among the concerns that some hold on the current system, one preeminent one is that 'its not what you know but who you know' that matters; namely, that established scientists have an easier time garnering the editor's attention and that they tend to give more favourable reviews of each other's work, while smaller upcoming labs struggle to publish. This worry seems to apply at both the editorial pre-screening stage and during the review process itself. We do not accept the alleged bias of the editorial pre-screening towards more established researchers. To avoid possible bias, we habitually employ multiple referees drafted from a wide pool of complementary scientific expertise, geographical locations and indeed affiliations. We also consciously balance the expertise of established leaders in the field with upcoming stars who often bring critical and fresh opinions to the process. As an integral part of this, we try to keep in touch with the research community through both lab visits and attendance at conferences. One idea aimed at circumventing such hypothetical biases is to have manuscripts assessed 'blind', without the authors' names on the submission. While interesting in principle, this is not a constructive solution as it is almost always obvious who authored a report based on presentations at meetings, previous publications, and even the citations in the manuscript. A more helpful approach to avoid potential bias is for authors to specify researchers who they feel might have a conflict of interest; we will honour such exclusions provided the number is within reason.

On the flip side, there is some concern that a researcher might agree to review a manuscript, despite the fact that they have an axe to grind and cannot provide an impartial review. This is unfortunately beyond our control, and we rely on the self-regulation of the community to ensure this does not happen. To avoid this problem, it has been suggested that reviewers should be asked to disclose their identity to authors, ensuring that the review process is open and constructive. Although we will disclose a referee's identity at his/her request, we do not favour this as a general policy, as it may prevent researchers from expressing a candid opinion. Moreover, it may work against less established researchers, who might succumb to a real or perceived feeling of intimidation by an established author and be reluctant to express their concerns, even if they are well founded, for fear that their next grant proposal will be rejected outright. Unfortunately, there will always be perceived or real 'hidden agendas' of potential favouritism.

Finally, an option explored by several journals is to publish referee reports alongside the accepted paper, or, even more radically, to disclose referee identity with the published report. While we do not favour the second option, we appreciate the transparency of the former and would welcome your feedback on this issue. One thing to consider is that obviously most aggravation arises from rejected manuscripts and neither option would address this. For now, enterprises such as 'Faculty of a 1000' (http://www.facultyof1000.com/) should go some way towards open evaluation.

Another discussed approach is to avoid peer review altogether and allow the piece to be judged over time, by the comments deposited by other researchers in the field and by the citation rate of a paper (an approach more established in physics circles). Sheer volume of publication restricts implementation to web-based publication. Although certainly conceptually enticing, in our view the sheer volume of data that would be published would make it near impossible for researchers to navigate the literature, let alone have time to provide feedback on the study.

We feel that the current multilevel filtering process provided by cell biology journals as a whole continues to sieve through the literature successfully and, although by no means perfect, is a fair approach. Nevertheless, we hope it will continue to evolve to meet your needs, and welcome correspondence on this issue. 